\title{
Design and Simulation of Insert to Measure Dynamic Sliding Friction in a Split-Hopkinson Pressure Bar
}

\author{
Virginia Euser $^{1 *}$, Austin Goodbody ${ }^{1}$, Clarissa Yablinsky $^{1}$, Nicholas Denissen ${ }^{1}$, and \\ Benjamin Morrow ${ }^{1}$ \\ ${ }^{1}$ Los Alamos National Laboratory, P.O. Box 1663, Los Alamos, NM 87544, USA
}

\begin{abstract}
Interfacial friction is a key aspect to understanding and modelling dynamic processes in which materials interact. However, friction is a complex phenomenon that depends on a multitude of factors, including sliding velocity. Understanding how friction behavior changes as a function of sliding rate is thus crucial for accurately simulating dynamic processes. Recent literature has shown that the split-Hopkinson pressure bar can be adapted for friction measurements associated with high sliding rates. The present work introduces an insert designed to be transferrable between a quasi-static load frame and a compression split-Hopkinson bar, enabling friction measurements across a wide range of sliding velocities $\left(10^{-4}-20\right.$ $\mathrm{m} / \mathrm{s}$ ). Here, the split-Hopkinson pressure bar setup is modelled using a multiphysics research code (FLAG), developed at Los Alamos National Laboratory (LANL), to identify and reduce potential issues in the configuration prior to experimental implementation.
\end{abstract}

\section{Introduction}

Interfacial friction significantly affects the behavior of dynamic systems in which materials interact. Friction is a complex phenomenon that can be a function of numerous parameters, including sliding velocity. Thus, to accurately model dynamic processes that occur at a range of rates, friction behavior must be understood over a multitude of sliding velocity regimes. The split-Hopkinson pressure bar (SHPB) is an experimental apparatus that allows for the measurement of material properties at relatively high strain rates, ranging from $10^{2}-10^{4} \mathrm{~s}^{-1}$ [1], [2]. Conventionally, the SHPB is used to measure material properties through direct, high rate compression. However, several adaptations of the SHPB have been designed to allow for the measurement of dynamic friction at relatively high sliding rates. This is typically achieved by replacing the traditional sample with a fixture that translates the strain pulse (compression, torsion, or tension) into sliding between two interfaces. The force measured in the transmitted bar is assumed to be solely associated with the friction force between the two sliding interfaces. The friction coefficient is then calculated from the measured friction force and known applied normal force.

* Corresponding author: ginny.euser@lanl.gov 
Torsion SHPBs have been utilized in numerous studies to measure dynamic friction [3][8], where sliding occurs between two nested tubular specimens that are placed between the incident and transmitted bars. Philippon [9] and others [10]-[14] coupled a gas gun and dynamometer ring to measure dynamic friction. Here, the gas gun is used to transfer a compressive force directly to a mobile specimen and the normal force is monitored using a dynamometer ring. Both Durand et al [15] and Bragov et al [16] used a compression SHPB to measure the friction between two nested cylindrical samples. More recently, Sanborn et al [17], [18] developed an experimental setup in which friction was measured utilizing a SHPB in tension.

The present work introduces a new insert designed to be transferrable between a quasistatic load frame and a compression split-Hopkinson bar, enabling friction measurements across a wide range of sliding velocities $\left(10^{-4}-20 \mathrm{~m} / \mathrm{s}\right)$. The experimental setup is modelled here using FLAG, a multi-physics research code developed at Los Alamos National Laboratory [19], [20]. FLAG is an explicit, finite-volume, staggered-grid, Arbitrary Lagrangian/Eulerian multi-physics code suitable for modeling solid material phenomena across a wide range of strain rates [21]. This work uses purely Lagrangian hydrodynamics with a general contact-surface algorithm that includes frictional forces on the sample interface [22]. Through modelling, this work strives to identify and reduce any potential issues inherent to the experimental design prior to experimental implementation. The model establishes a framework to rapidly explore alterations in the fixture design. In the future, the model developed here will be validated through experimental tests.

\section{Procedures}

A schematic of the experimental friction fixture design is shown in Figure 1, in which the general concept is modelled after a tensile configuration presented by Sanborn et al [17], [18]. The left side of the assembly is displaced through an applied load (quasi-static), or the propagation of an elastic wave (SHPB), and results in sliding between materials 1 and 2 . Normal force is applied via set screws and is monitored by a piezo film sensor located between the backing platen and specimen.

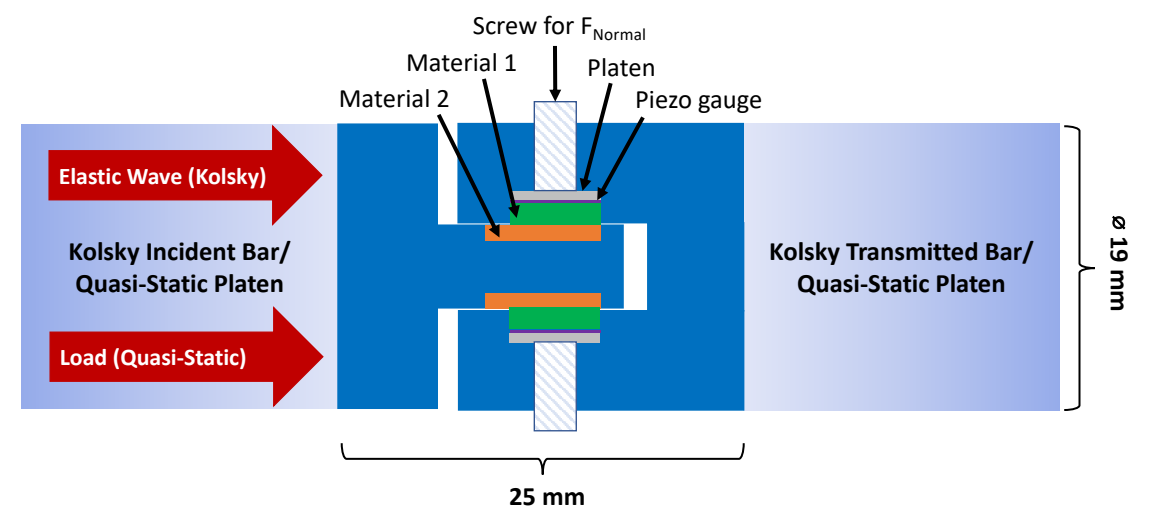

Fig. 1. Illustration of experimental friction fixture design to be incorporated into a compression SHPB.

The present work simulates the behavior of the experimental friction assembly within a full SHPB configuration. Figure 2 illustrates the simulation setup, in which the interaction between the striker, incident bar, friction fixture, and transmitted bar are modelled. The friction assembly is simplified compared to the intended experimental design (Figure 1) by neglecting the presence of the set screw, platen, and piezo sensor. The simulations presented here assume 2-D axisymmetry, as it provides the best balance of computational cost and 
model fidelity. Although the experimental loading configuration is planar, axisymmetry is assumed to more accurately capture wave speeds in 2-D. Given the axisymmetric nature of the simulations, the contact area between the two materials will differ between the experimental configuration and the model, but is normalized during the analysis process. A pressure boundary condition is applied to the outer surface of the housing sample (green) to simulate normal force applied through the set screw. Friction is implemented at the interface between the housing and rod samples (green and orange, respectively) using a classical coulomb friction model. For all simulations, the static and kinetic friction coefficients are equal. Table 1 provides additional geometry and material information relevant to the simulations.

Velocity, pressure, displacement, and density are collected using tracers positioned at the mid-length of the incident and transmitted bars. Strain is calculated based on displacement, where two tracers are placed at the mid-length of each bar and separated by some relatively small distance $(l)$. Here, the tracers are $1 \mathrm{~mm}$ apart, and the midpoint between the tracers correspond to likely locations of experimental strain gauges (see Table 1). The change in the distance between the two tracers relative to the original distance is translated into strain by

$$
e=\frac{\Delta l}{l_{o}}
$$

Friction force is calculated from the transmitted strain wave via

$$
F_{\text {friction }}=A_{\text {rod }} E \varepsilon_{\mathrm{t}}
$$

where $A_{\text {rod }}$ is the cross-sectional area of the transmitted rod, $E$ is the elastic modulus of the $\operatorname{rod}$ material, and $\varepsilon_{\mathrm{t}}$ is the transmitted strain. The coefficient of friction is then calculated knowing

$$
\mu=\frac{F_{\text {friction }}}{F_{\text {normal }}}
$$

where $F_{\text {friction }}$ is the friction force derived from Equation 2 and $F_{\text {normal }}$ is the normal force applied through the pressure boundary condition. The normal force is calculated from the known contact area and applied pressure.

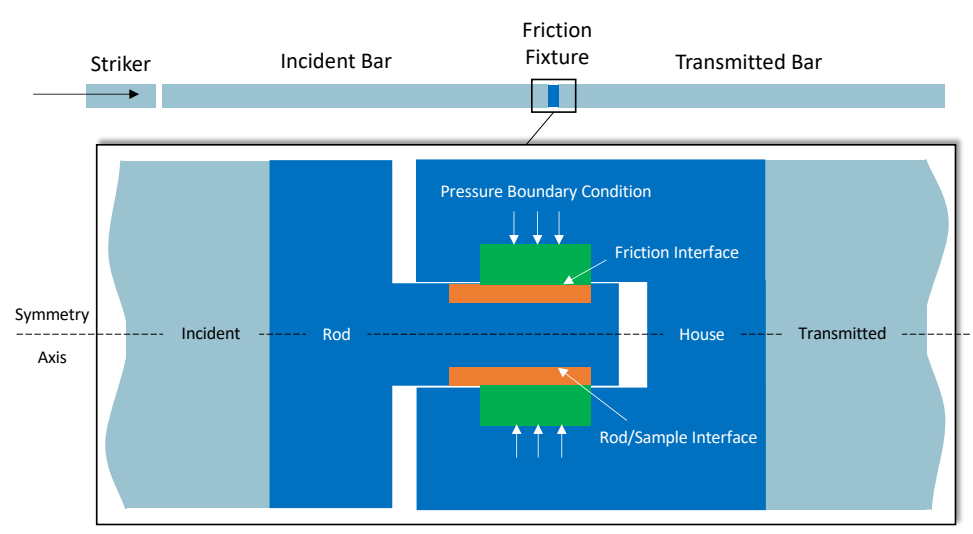

Fig. 2. Illustration of FLAG simulation setup highlighting key boundaries and interfaces.

Table 1. Simulation Parameters

\begin{tabular}{|c|c|}
\hline Incident/Transmitted Bar Material & 350 Maraging Steel \\
\hline Friction Fixture Material & 350 Maraging Steel \\
\hline Sample Material & Al 6061-T6 \\
\hline Striker Length (mm) & 150 \\
\hline
\end{tabular}




\begin{tabular}{|c|c|}
\hline Striker Velocity (m/s) & 15 \\
\hline Incident/Transmitted Bar Length (mm) & 762 \\
\hline Incident/Transmitted Bar Diameter (mm) & 19.05 \\
\hline $\begin{array}{c}\text { Midpoint Location of Tracers Used for Strain } \\
\text { Calculation (mm) }\end{array}$ & 381 \\
\hline Normal Pressure (MPa) & $25-125 \mathrm{MPa}$ \\
\hline
\end{tabular}

\section{Results}

Preliminary studies were conducted assuming two different rod/sample boundary conditions: free and fixed. The free condition allows unconstrained movement of the sample in the $\mathrm{x}$ direction within the rod slot. In contrast, the fixed condition stitches the rod and sample together, effectively limiting movement in the x-direction at the rod/sample interface. These conditions were designed to reflect two possible experimental configurations in which the sample could be bonded to the rod or allowed to move independently. Simulations were run over a range of prescribed friction coefficients ( 0.1 to 1$)$ and normal pressures $(25-100$ $\mathrm{MPa}$ ). Figure 3 depicts the simulation results for the free and fixed conditions assuming a static and kinetic friction coefficient of 0.6 . The sample response is significantly affected by the boundary condition at the rod/sample interface, where the free condition results in minimal sliding at the friction interface and significant sample deformation. Conversely, the fixed condition primarily exhibits sliding at the friction interface and shows little to no sample deformation. Thus, affixing the sample to the rod encourages sliding at the friction interface, a pivotal aspect of the intended fixture design. By simulating the system, it is evident that bonding the sample and rod together is necessary to achieve the desired results, and will thus be adopted for future experiments (and all subsequently presented simulations).

(a)
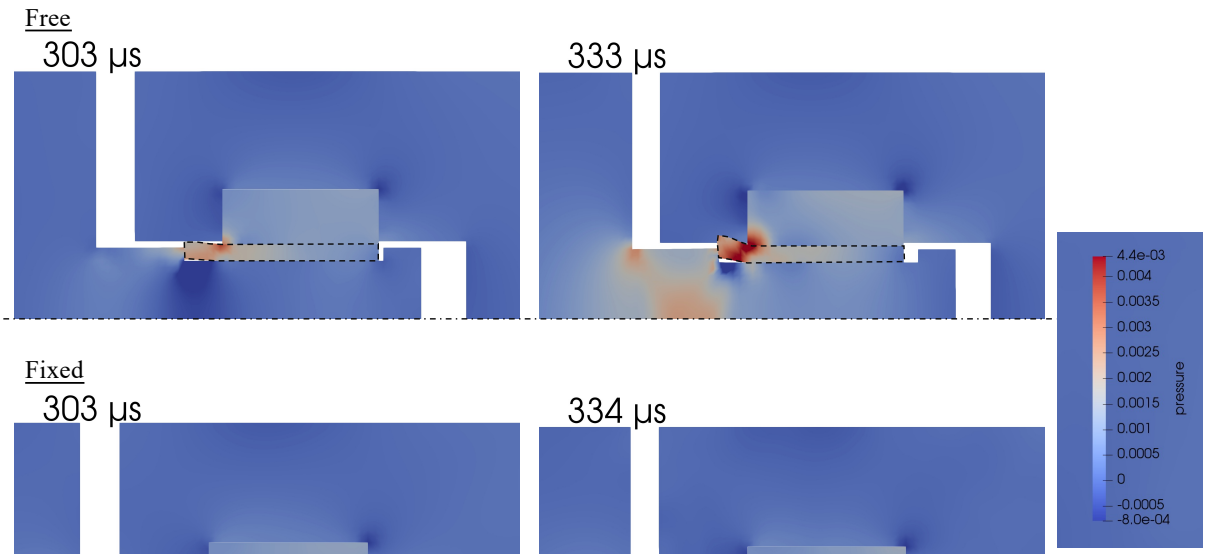

(b)
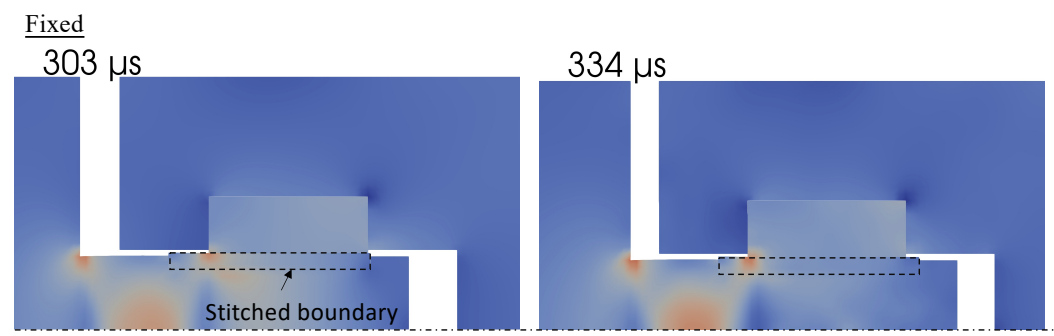

Fig. 3. Sample behaviour associated with a (a) free and (b) fixed sample/rod interface for friction conditions $\mu_{\mathrm{k}}=\mu_{\mathrm{s}}=0.6$ and a normal applied pressure of $100 \mathrm{MPa}$. The illustrated pressure scale is represented in MBar.

Figure 4(a) shows an example of the strain results assuming a normal pressure of 100 $\mathrm{MPa}$ and a friction coefficient of 1 . The recorded incident, reflected, and transmitted strain 
waves are qualitatively similar to what would be expected for a traditional compression SHPB. In this case, rather than using the strain wave response to deduce stress-strain behavior of a compression sample, the transmitted wave behavior is utilized to infer friction conditions at the probed friction interface. Using Equations 2 and 3, the transmitted strain is converted into friction coefficient to produce Figure 4(b). The friction coefficient peak oscillates about a value close to the friction coefficient dictated at the interface, 1 .

Figure 5(a) displays the average friction coefficient results associated with a normal pressure of $100 \mathrm{MPa}$. Here, friction coefficient derived from the transmitted strain is shown as a function of the friction value set at the interface. Friction coefficients were quantified by averaging the values at the plateau of the friction coefficient peak (Figure 4(b)), and error was calculated as one standard deviation of the averaged values. Ideally, the friction coefficient derived from the transmitted pulse would correlate to the friction coefficient dictated at the friction interface. Compared to an idealized 1:1 linear relationship, there is some deviation between the calculated and set friction coefficients shown in Figure 5(a), although the overall agreement is good.

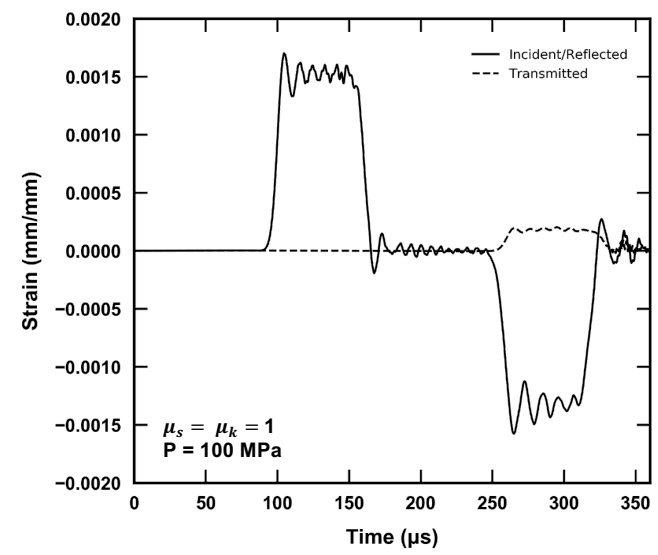

(a)

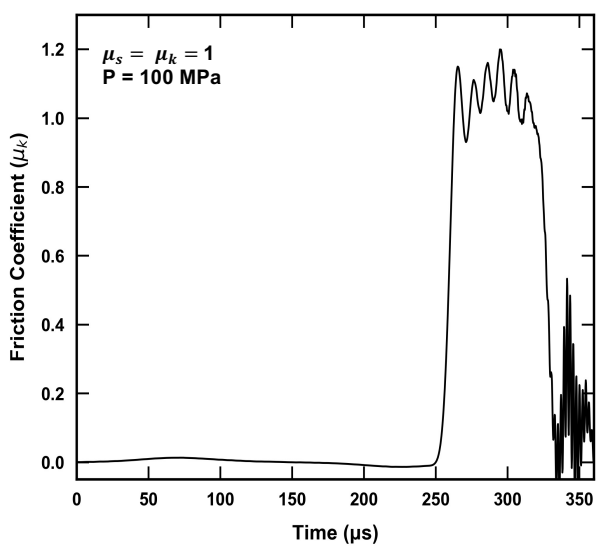

(b)

Fig. 4. (a) Simulation strain results for friction conditions $\mu_{\mathrm{k}}=\mu_{\mathrm{s}}=1$ and a normal applied pressure of $100 \mathrm{MPa}$. (b) Corresponding friction coefficient calculated from the transmitted strain in (a).

Figure 5(b) shows the effect of varying applied normal pressure on the relationship between the set and detected friction coefficients. Below a set friction coefficient of 0.6 , the results are close to the idealized 1:1 relationship and show little variation as a function of applied pressure. According to classical laws of friction, the friction coefficient is independent of applied load, and some experimental studies have found this to be true [23]. Others [23]-[25] have reported systematic variations in friction coefficient as a function of normal force, although this behavior is attributed to mechanisms and phenomena that are not included in the present model. Thus, varying normal pressure is anticipated to produce consistent friction behavior in the present simulations, as is observed below a set friction coefficient of 0.6 . Above 0.6 , lower normal pressures exhibit a significant deviation from the 1:1 idealized behavior, and substantial discrepancies are observed across the explored normal pressures. The source of this behavior is not currently known, although may be associated with finite deformation effects or other issues with the model. Experimental tests are presently being pursued to compare to the simulated results prior to developing the model further. 


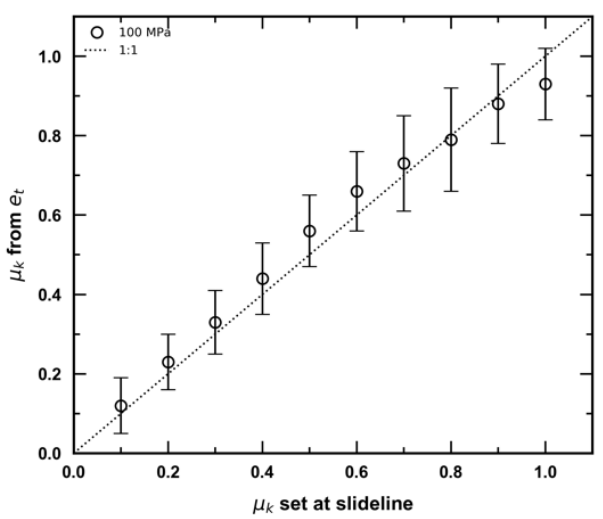

(a)

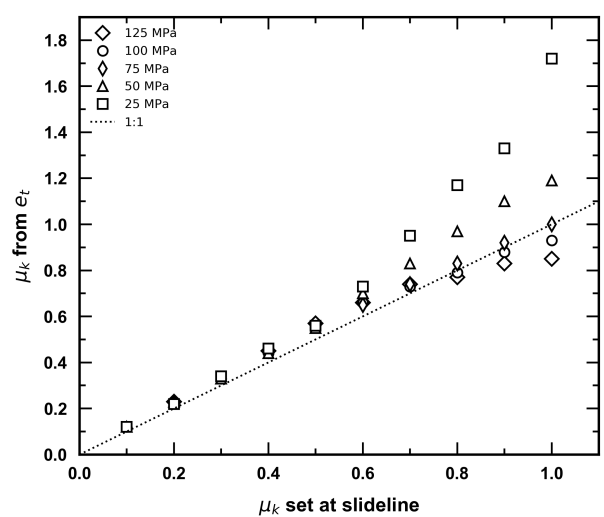

(b)

Fig. 5. Comparison of the friction coefficient dictated at the slide interface (slideline) and the average friction coefficient calculated from the resulting transmitted strain for (a) a normal pressure of 100 $\mathrm{MPa}$ and (b) normal pressures ranging from 25 to $125 \mathrm{MPa}$. Error is associated with one standard deviation of the averaged values and the dotted line represents a 1:1 linear relationship.

\section{Discussion and Conclusions}

A new experimental configuration for measuring dynamic friction using a compression SHPB was modelled with a multi-physics code, FLAG. The simulations revealed the need for a key design change in order to promote sample sliding at the friction interface. Without bonding the inner sample to the rod fixture, the majority of sliding occurs at the sample/fixture interface rather than the sample/sample interface of interest. This can be mitigated by affixing the inner sample to the rod fixture, thus promoting sliding between the two samples. Sliding between samples was achieved in the simulations by stitching the sample/rod interface together, and will be mimicked in the experimental setup by bonding the inner sample to the rod fixture.

The strain and friction coefficient results offer a promising working model that is particularly valuable for friction coefficients up to 0.6. The developed model can be used to rapidly prototype various fixture configurations prior to experimental implementation, as was demonstrated through the exploration of the fixed vs free rod/sample interface conditions. The behavior observed above a friction coefficient of 0.6 warrants further study and presents an opportunity to refine the established model. Future work will involve validating the model against experimental friction measurements.

This work was conducted under the guidance of the US Department of Energy and was supported by Laboratory Directed Research and Development at Los Alamos National Laboratory.

\section{References}

[1] W. Chen and B. Song, Split Hopkinson (Kolsky) Bar. Boston, MA: Springer US, 2011.

[2] G. T. Gray III, "Classic Split-Hopkinson Pressure Bar Testing," in Mechanical Testing and Evaluation, H. Kuhn and D. Medlin, Eds. ASM International, 2000, pp. $462-476$. 
[3] H. D. Espinosa, A. J. Patanella, and M. Fischer, "Dynamic Friction Measurements at Sliding Velocities Representative of High-Speed Machining Processes," J. Tribol., vol. 122, no. 4, pp. 834-848, Oct. 2000, doi: 10.1115/1.1310331.

[4] H. D. Espinosa, A. Patanella, and M. Fischer, "A novel dynamic friction experiment using a modified kolsky bar apparatus," Exp. Mech., vol. 40, no. 2, pp. 138-153, Jun. 2000, doi: 10.1007/BF02325039.

[5] S. Rajagopalan and V. Prakash, "A modified torsional kolsky bar for investigating dynamic friction," Exp. Mech., vol. 39, no. 4, pp. 295-303, Dec. 1999, doi: 10.1007/BF02329808.

[6] F. Yuan and V. Prakash, "Use of a modified torsional Kolsky bar to study frictional slip resistance in rock-analog materials at coseismic slip rates," Int. J. Solids Struct., vol. 45, no. 14-15, pp. 4247-4263, Jul. 2008, doi: 10.1016/j.ijsolstr.2008.03.012.

[7] B. Rodrigues, "Dynamic Frictional Response of Granular Materials Under Seismically Relevant Conditions Using a Novel Torsional Kolsky Bar Apparatus," Masters of Science, Case Western Reserve, 2017.

[8] K. Ogawa, "Impact friction test method by applying stress wave," Exp. Mech., vol. 37, no. 4, pp. 398-402, Dec. 1997, doi: 10.1007/BF02317304.

[9] S. Philippon, G. Sutter, and A. Molinari, "An experimental study of friction at high sliding velocities," Wear, vol. 257, no. 7-8, pp. 777-784, Oct. 2004, doi: 10.1016/j.wear.2004.03.017.

[10] J. J. Arnoux, G. Sutter, G. List, and A. Molinari, "Friction Experiments for Dynamical Coefficient Measurement," Adv. Tribol., vol. 2011, pp. 1-6, 2011, doi: 10.1155/2011/613581.

[11] A. Lodygowski, L. Faure, G. Z. Voyiadjis, and S. Philippon, "Dry Sliding Friction Experiments at Elevated Velocities: Dry Sliding Friction Experiments," Strain, vol. 47, pp. 436-453, Dec. 2011, doi: 10.1111/j.1475-1305.2010.00785.x.

[12] G. List, G. Sutter, J. J. Arnoux, and A. Molinari, "Study of friction and wear mechanisms at high sliding speed," Mech. Mater., vol. 80, pp. 246-254, Jan. 2015, doi: 10.1016/j.mechmat.2014.04.011.

[13] G. List, G. Sutter, and J. J. Arnoux, "Analysis of the high speed sliding interaction between titanium alloy and tantalum," Wear, vol. 301, no. 1-2, pp. 663-670, Apr. 2013, doi: 10.1016/j.wear.2012.11.070.

[14] G. Sutter and N. Ranc, "Flash temperature measurement during dry friction process at high sliding speed," Wear, vol. 268, no. 11-12, pp. 1237-1242, May 2010, doi: 10.1016/j.wear.2010.01.019.

[15] B. Durand, F. Delvare, P. Bailly, and D. Picart, "Friction Between Steel and a Confined Inert Material Representative of Explosives Under Severe Loadings," Exp. Mech., vol. 54, no. 7, pp. 1293-1303, Sep. 2014, doi: 10.1007/s11340-014-9885-z.

[16] A. M. Bragov, A. Yu. Konstantinov, and A. K. Lomunov, "Determining dynamic friction using a modified Kolsky method," Tech. Phys. Lett., vol. 34, no. 5, pp. 439440, May 2008, doi: 10.1134/S1063785008050234.

[17] B. Sanborn, B. Song, and E. E. Nishida, "Development of a New Method to Investigate Dynamic Friction Behavior of Metallic Materials Using a Kolsky Tension Bar.," SAND2017-11985, 1596207, Nov. 2017. doi: 10.2172/1596207.

[18] B. Sanborn, B. Song, and E. Nishida, "Development of a New Method to Investigate the Dynamic Friction Behavior of Interfaces Using a Kolsky Tension Bar," Exp. Mech., vol. 58, no. 2, pp. 335-342, Feb. 2018, doi: 10.1007/s11340-017-0350-7.

[19] D. E. Burton, "Connectivity Structures and Differencing Techniques for StaggeredGrid Free-Lagrange Hydrodynamics,” New Brunswick, New Jersey, 1992.

[20] D. E. Burton, "Consistent finite-volume discretization of hydrodynamic conservation laws for unstructured grids," Las Vegas, NV, 1994. 
[21] E. J. Caramana, D. E. Burton, M. J. Shashkov, and P. P. Whalen, "The Construction of Compatible Hydrodynamics Algorithms Utilizing Conservation of Total Energy," J. Comput. Phys., vol. 146, pp. 227-262, 1998.

[22] M. T. Bement and M. A. Kenamond, "Slideline modeling in the FLAG hydrocode," No. LA-UR-11-04993, 2011.

[23] D. F. P. Bowden and D. Tabor, "Mechanism of Metallic Friction," p. 3, 1942.

[24] J. R. Whitehead, "Surface deformation and friction of metals at light loads," p. 20, 1950.

[25] E. Rabinowicz, "Friction coefficients of noble metals over a range of loads," Wear, vol. 159, no. 1, pp. 89-94, Nov. 1992, doi: 10.1016/0043-1648(92)90289-K. 